\title{
Splenic Injury after Screening Colonoscopy; Could that Happen Twice? An Unusual Case Report
}

\author{
Christopoulos $\mathrm{P}^{*}$, Rajveer TS, Rajput I and Ahmed T
}

Airedale General Hospital, Surgical Department, Skipton Rd, Steeton, BD20 6TD, United Kingdom

*Corresponding author: Christopoulos P, MD, MSc, 28 Teal House, Skipton Rd, BD208US, United Kingdom, Tel: +447474057275, E-mail: petchristopoulos@gmail.com

Citation: Christopoulos P, Rajveer TS, Rajput I, Ahmed T (2017) Splenic Injury after Screening Colonoscopy; Could that Happen Twice? An Unusual Case Report. J Case Rep Stud 5(4): 401. doi: 10.15744/2348-9820.5.401

Received Date: May 16, 2017 Accepted Date: August 28, 2017 Published Date: August 30, 2017

\begin{abstract}
Background: Iatrogenic splenic injury is a known but rare complication of colonoscopic procedures. Our department describes a very interesting case of the same complication that occurred twice on the same patient.

Description of the Case: The patient had an elective colonoscopy for microcytic anemia and shortly after the endoscopic procedure she developed excessive abdominal pain and had a syncope episode. She gradually became hemodynamically unstable and needed proper resuscitation. An abdominal tomography scan performed which surprisingly demonstrated active intra-abdominal bleeding pointing a ruptured spleen as the source of the hemorrhage. This finding surprised us given the fact that her past surgical history included a previous splenectomy for the same complication 14 years ago. The patient underwent an emergency laparotomy for control of the hemorrhage and a splenectomy of an actively bleeding spleen was performed. Thorough investigation of the abdominal cavity was negative for residual splenic tissue. The patient was discharged after four days of an uncomplicated post-surgical recovery.
\end{abstract}

Conclusion: We postulate that there was either a splenosis, or a splenunculus. There is a possibility that the previous splenectomy was partial. To the best of our knowledge this is the first reported case of the same rare complication that happened twice to the same patient.

Keywords: Splenic Injury; Intra-Abdominal Bleeding; Colonoscopy; Splenosis; Splenunculus

\section{Introduction}

The number of colonoscopies being performed globally has increased significantly over the past four decades and the procedure is now the mainstay investigation for benign and malignant colonic pathologies. Iatrogenic splenic injury is a known but rare complication of colonoscopic procedures. It was first reported and highlighted as an acute complication of the procedure by Wherry and Zehner [1] in 1974 in a case series of 247 polypectomies. Since the 1970's, approximately 68 cases of splenic injury secondary to colonoscopy have been published in the literature [2]. It has been reported to have an incidence between $0.00005 \%$ and $0.017 \%$ with a significant mortality rate of 5\% [3]. Cases are noted to be potentially underreported due to publication bias, insufficiency of specific codes and disinclination to publish morbidity and mortality data [4]. To the best of our knowledge, this is the first reported case to describe twice a splenic injury after colonoscopy to the same patient, who has been treated both times with emergency open splenectomy.

\section{Case report}

Our centre reports a very interesting case of a 72 year old lady who had the same complication twice within 14 years. She has a past medical history of anxiety disorder, iron deficiency anaemia and hypothyroidism. She did not mention any related medical or surgical family history but her surgical history included a splenectomy for iatrogenic splenic rupture following a colonoscopy 14 years earlier. Unfortunately, we could not retrieve her previous medical notes since the operation took place in a different hospital. A month before the acute admission she had a Computer Tomography (CT) colonography scan investigating iron deficiency anemia and some non-specific abdominal symptoms, which demonstrated a $7 \mathrm{~mm}$ flat cecal polyp. She subsequently underwent a colonoscopy. In the two three hours after the colonoscopy, while in recovery, the patient developed unremitting left upper quadrant pain and had symptoms of syncope and hypotension. The patient was admitted to the hospital directly from the endoscopy unit under the care of the medical team. Hemoglobin $(\mathrm{Hb})$ on admission had fallen to $91 \mathrm{~g} / \mathrm{L}$. Four hours after admission the surgical team on call was asked to review the patient due to persistence of the abdominal pain and hypotension. An urgent CT abdomen/ 
pelvis scan was arranged. The scan revealed a large perisplenic haematoma with active splenic extravasation of contrast (Figure 1). Images demonstrated also large amount of free blood in the abdomen and the pelvis (Figure $2 \mathrm{~A}$ and $\mathrm{B}$ ). Repeated $\mathrm{Hb}$ at that time was $67 \mathrm{~g} / \mathrm{L}$. The decision for an emergency laparotomy was taken within 6 hours after admission and the patient finally was in the operating room within an hour after the consent while she was having haemorrhage protocol resuscitation and being hemodynamically stable.

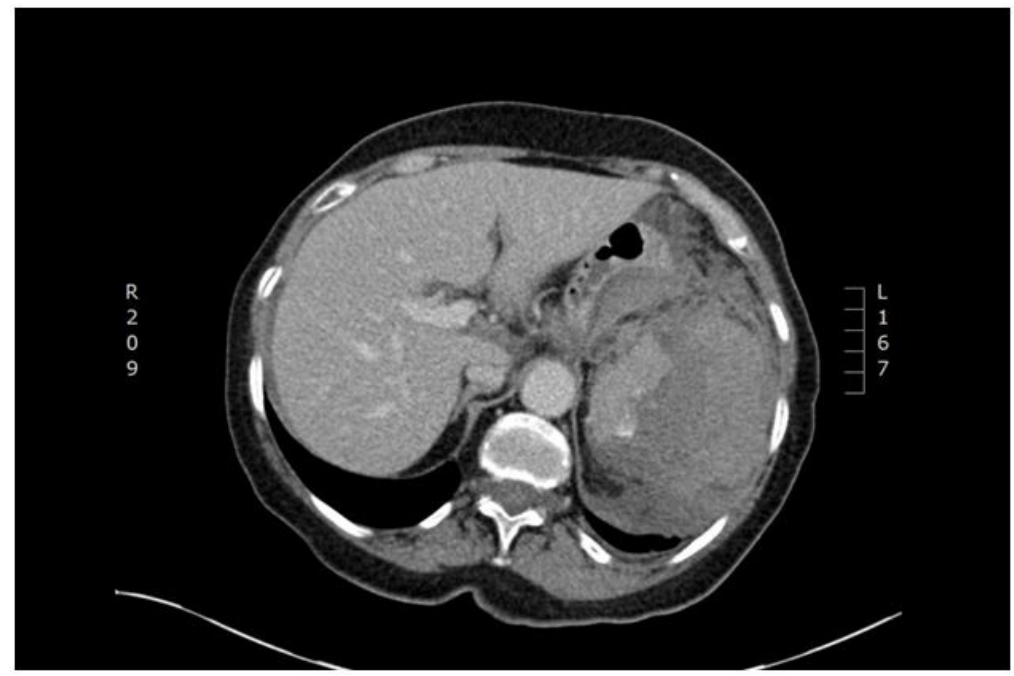

Figure 1: Pre-operative CT of the abdomen showing the active bleeding from the spleen and the peri-splenic hematoma

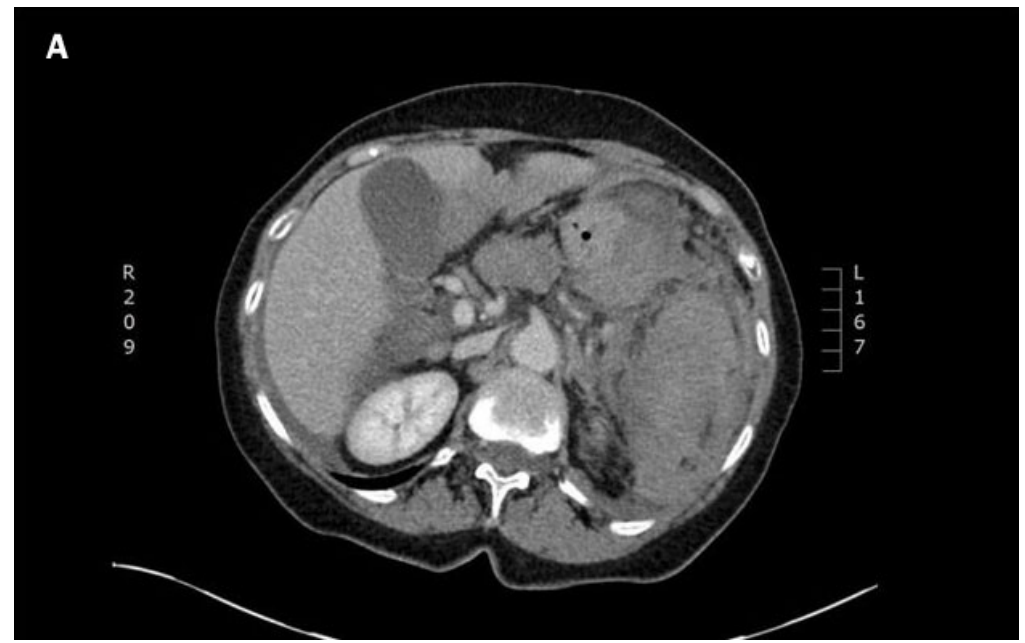

$\mathbf{B}$

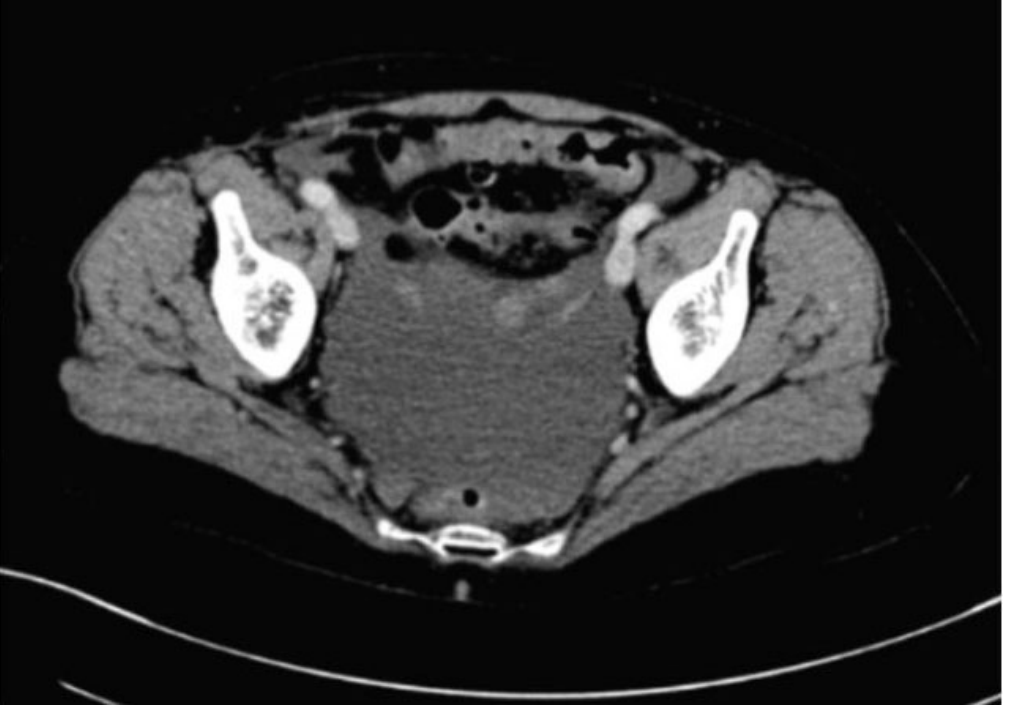

Figure 2A and B: Images demonstrating the haemoperitoneum (free blood around the liver and in the pelvis) 
Intraoperative findings were consistent with splenic hemorrhage. Subsequently we performed a splenectomy (Figure 3). Thorough investigation of the abdominal cavity was negative for residual splenic tissue. Histology confirmed normal ruptured splenic tissue with hematoma. The patient had an uneventful post-surgical course and discharged four days after. 6 weeks post-operation outpatient follow-up was unremarkable.

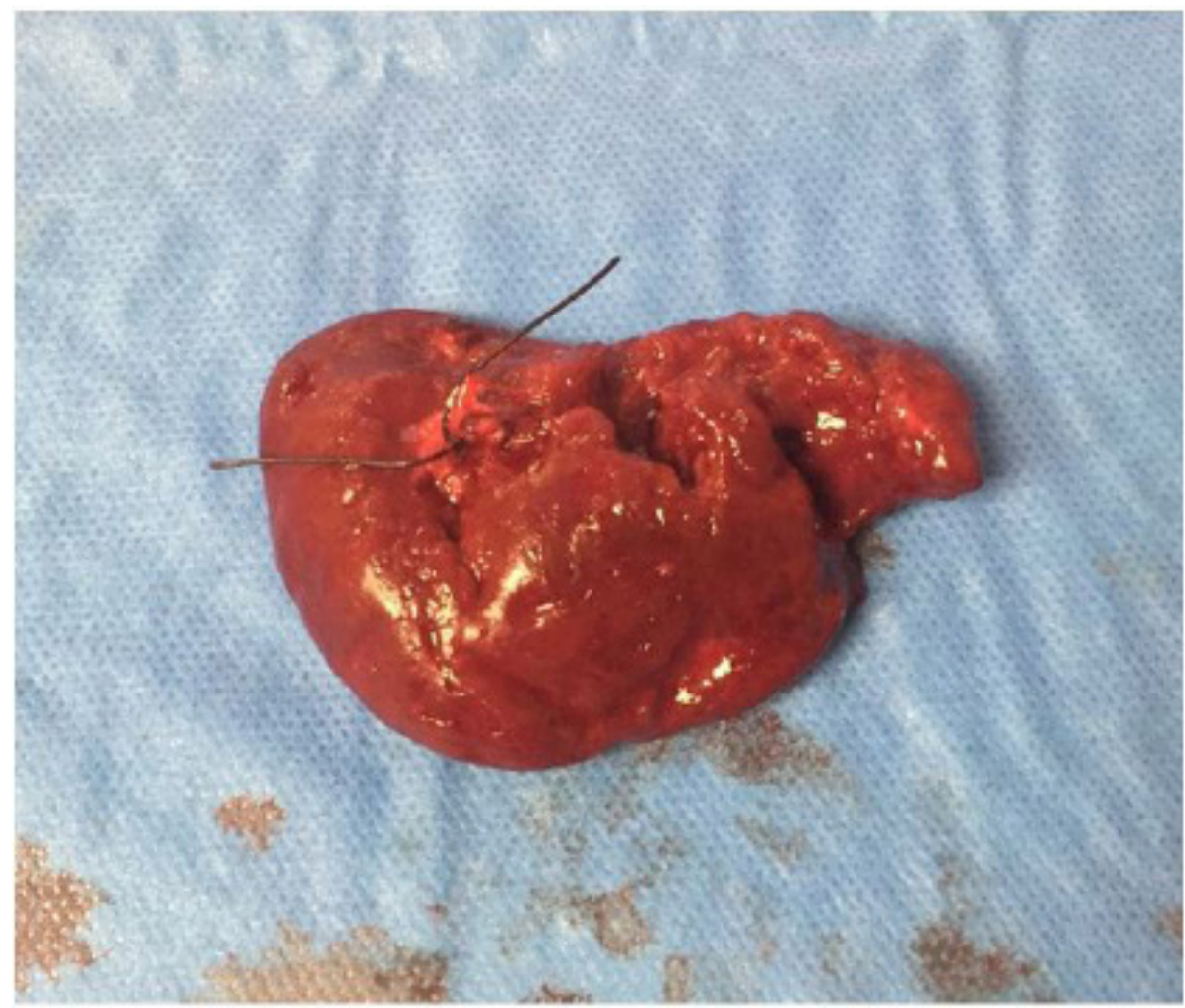

Figure 3: The removed spleen measured $7 \mathrm{~cm}$ x $5 \mathrm{~cm}$ x $2 \mathrm{~cm}$ with obvious rapture injury at the visceral surface close to the hilum

\section{Discussion}

Severe abdominal pain within 24 hours of having a colonoscopy warrants prompt investigation and a high index of suspicion of possible complications including hemorrhage $(0.001 \%-2 \%)$ and colonic perforation $(0.1-0.267 \%)[5,6]$. Iatrogenic splenic injury during colonoscopy is a rare but reported complication which can cause substantial risk of morbidity and mortality. A pertinent, systematic review reported the most likely mechanisms of splenic injury during the procedure were; looping of instruments, difficult intubation, traction on the splenocolic ligament and presence of a large polyp in the splenic flexure [5].

Although splenic trauma can occur in fit and healthy adults undergoing colonoscopy it has been suggested that intra-abdominal adhesions due to previous surgery escalate the risk of extra-capsular forces on the spleen thus pre-disposing to an increased risk of capsular avulsion [5,7].

The diagnosis is likely to be delayed as post procedural pain is attributed to more common etiologies such as air insufflation and resultant colonic distension. The symptoms may be masked due to sedation, impaired mental status and analgesia.

Management of patients with splenic trauma is dependent on both their hemodynamic stability and the severity of splenic injury. Splenic injuries can be graded using the American Association for the Surgery of Trauma splenic injury classification from Grade I to $\mathrm{V}$ depending on the depth and level of the laceration identified on CT scan and the size and position of the subsequent haematoma. Grades I-III injuries are potentially suitable for conservative management if the patient remains haemodynamically stable. Due to the extent of injury, grades IV and V carry an increased morbidity and mortality rate and are less likely to be managed conservatively. Frequently, these warrant prompt exploration of the abdominal cavity and surgical control of the bleeding. In recent years with the significant advancement of interventional radiological techniques, splenic injuries can be successfully managed with angioembolization $[5,8]$. Our patient however, was hemodynamically unstable and not suitable for transfer to the local interventional radiology department therefore we decided to proceed with an emergency laparotomy.

The case described in this report, is exceptionally unusual as this unfortunate patient had already undergone a splenectomy for the exact same complication fourteen years previously. We were obviously surprised by both the CT and intraoperative findings given her previous splenectomy.

Upon review on the literature three theories can support our findings in this case. 
Firstly residual splenic tissue auto-transplanted and grew to function as a new splenic unit during the first splenic injury; known as splenosis and its blood supply is by new formed artery that penetrates the spleen. The process of splenic auto-transplantation has been documented as early as 1976 [9]. Splenosis can occur in a variety of locations within the abdomen and cases have even been reported of thoracic splenosis from concomitant splenic and diaphragmatic rupture [9].

The second potential explanation is the hypertrophy of a congenital, accessory spleen, also called a splenunculus which may have grown to take on the function of the absent spleen. In embryological development, splenunculi are formulated from ectopic splenic tissue and arise from the left side of the dorsal mesogastrium and their blood supply is by branches of the splenic artery. The incidence of splenunculi has been quoted as high at 30\% of patients at autopsy [10]. Once a splenectomy, is performed the splenunculus can grow and take on the full role of the original spleen and can potentially grow to the full size of the original organ.

The third potential theory is that there was an incomplete splenectomy at the time of the first emergency laparotomy. This theory suggests that during an emergency splenectomy, the surgeon ligating the hilum vessels can leave behind some splenic tissue at the hilum and that tissue could regenerate to a neoformed spleen later at some degree. Unfortunately, we were unable to retrieve the past surgical record and histology report to obtain more details for the exact procedure [11]. However, given she was on long term antibiotic prophylaxis and the anatomy of our specimen we believe that this theory is the least likely for our case.

Regardless of which of the mentioned theories occurred, the patient had a second proven splenic rupture following her colonoscopy. To the best of our knowledge this is the first report of such an event.

\section{Conclusion}

Colonoscopy is the gold standard diagnostic tool for investigation of colonic pathology and overall it remains a safe procedure. However, ongoing post colonoscopy pain and physiological instability should trigger suspicions and demand prompt investigation.

\section{Acknowledgments}

The case has been presented as a poster presentation to the ACG 2016 Annual Scientific Meeting \& Postgraduate Course (American College of Gastroenterology), Las Vegas October 2016.

The authors declare there is no conflict of interest and we obtained a signed informed consent from the patient. Author contribution was shared between equally between all four authors.

\section{References}

1. Wherry DC, Zehner H (1974) Colonoscopy-fiberoptic endoscopic approach to the colon and polypectomy. Med Ann Dist Columbia 43: 189-92.

2. Sarhan M, Ramcharan A, Ponnapalli S (2009) Splenic Injury after Elective Colonoscopy. JSLS 13: 616-9.

3. Di Lecce F, Vigano P, Pilati S, Mantovani N, Togliani T, et al. (2007) Splenic rupture after colonoscopy. A case report and review of the literature. Chir Ital 59: 755-7.

4. Luebke T, Baldus SE, Holscher AH, Monig SP (2006) Splenic rupture: an unusual complication of colonoscopy. Surg Laparosc Endosc Percutan Tech 16: 351-4.

5. Ha JF, Minchin D (2009) Splenic injury in colonoscopy: a review. Int J Surg 7: 424-7.

6. Prowda JC, Trevisan SG, Lev-Toaff AS (2005) Splenic injury after colonoscopy: conservative management using CT. AJR Am J Roentgenol 185: 708-10.

7. Levine E, Wetzel LH (1987) Splenic trauma during colonoscopy. AJR Am J Roentgenol 149: 939-40.

8. Watson GA, Hoffman MK, Peitzman AB (2015) Nonoperative management of blunt splenic injury: what is new? Eur J Trauma Emerg Surg 41: 219-28.

9. Fleming CR, Dickson ER, Harrison EG Jr (1976) Splenosis: autotransplantation of splenic tissue. Am J Med 61: 414-9.

10. Mortelé KJ, Mortelé B, Silverman SG (2004) CT Features of the Accessory Spleen. AJR Am J Roentgenol 183: 1653-7.

11. Khan ZA, Dikki PE (2004) Return of a normal functioning spleen after traumatic splenectomy. J R Soc Med 97: $391-2$.

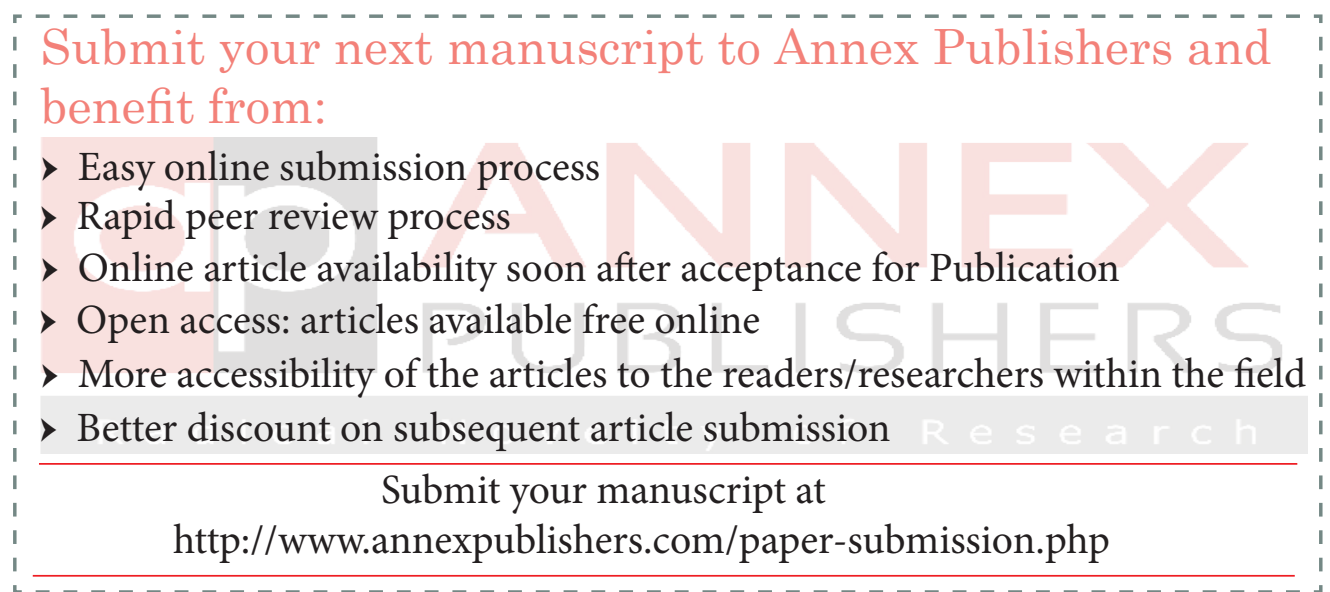

